\title{
BASIC PROPERTIES OF LOG CANONICAL CENTERS
}

\author{
FLORIN AMBRO
}

\begin{abstract}
We present the elementary properties of log canonical centers of log varieties.
\end{abstract}
\section{INTRODUCTION}

Log varieties and their log canonical centers provide a natural setting for inductive arguments in higher dimensional algebraic geometry. The prototype log variety is $\left(X, \sum_{i} b_{i} E_{i}\right)$, where $X$ is a nonsingular variety, the $E_{i}$ 's are nonsingular prime divisors intersecting transversely, and $b_{i} \in[0,1]$. If $b_{i}<1$ for all $i,\left(X, \sum_{i} b_{i} E_{i}\right)$ has so called Kawamata log terminal singularities, and it has no $\log$ canonical centers. In general, some of the $E_{i}$ 's will have coefficient one, and the connected components of their intersections are called $\log$ canonical centers. Further, let $C$ be a $\log$ canonical center which is a connected component of $\cap_{i \in J} E_{i}$, where $b_{i}=1$ for all $i \in J$. Then a successive application of the classical adjunction formula gives the log adjunction formula

$$
\left.\left(K_{X}+\sum_{i} b_{i} E_{i}\right)\right|_{C}=K_{C}+\left.\sum_{i \notin J} b_{i} E_{i}\right|_{C} .
$$

Creating log canonical centers, restricting to them by adjunction, and lifting sections via vanishing theorems - these are the three steps of a powerful technique for constructing sections of adjoint line bundles in characteristic zero, parallel to the $\mathrm{L}^{2}$-methods for singular hermitian metrics in complex geometry (see [11, 9, 15, 3] and [18, 7] for the algebraic and analytic side of the story, respectively).

In this note we present the elementary properties of log canonical centers. They are easy to see in our example above: its log canonical centers are nonsingular, finite in number, their intersections are unions of $\log$ canonical centers, and their unions have seminormal singularities. Most of these properties extend to the general case of log varieties with log canonical singularities, by Shokurov [17], Kollár [14], Ein-Lazarsfeld [8], Kawamata [12] and 2], under some mild extra hypotheses, and [4] in general. Since they seem to be obscured by the new terminology of quasi-log varieties in [4], we reproduce them here.

Theorem 1.1. Let $(X, B)$ be a log variety with log canonical singularities, defined over an algebraically closed field of characteristic zero. Then:

(1) $(X, B)$ has at most finitely many log canonical centers.

(2) An intersection of two log canonical centers is a union of log canonical centers.

(3) Any union of log canonical centers has seminormal singularities.

\footnotetext{
${ }^{1}$ The author is supported by a 21st Century COE Kyoto Mathematics Fellowship, and a JSPS Grantin-Aid No 17740011.

${ }^{2} 1991$ Mathematics Subject Classification. Primary: 14B05. Secondary: 14E30
} 
(4) Let $x \in \operatorname{LCS}(X, B)$ be a closed point. Then there is a unique minimal log canonical center $C_{x}$ passing through $x$, and $C_{x}$ is normal at $x$.

In previous approaches, when there exists $0 \leq B^{0} \leq B$ such that $\left(X, B^{0}\right)$ has Kawamata log terminal singularities, Theorem 1.1 follows from Kawamata-Viehweg's vanishing. The new idea is to use the log canonical version of Kollár's torsion freeness, instead of vanishing.

\section{LOG VARIETIES}

A $\log$ variety $(X, B)$ is a normal variety $X$ endowed with an effective $\mathbb{R}$-Weil divisor $B$ such that $K_{X}+B$ is $\mathbb{R}$-Cartier. We assume that $X$ is defined over an algebraically closed field $k$, of characteristic zero. The canonical divisor $K_{X}$ is defined as the Weil divisor $(\omega)$ of zeros and poles of a non-zero top rational differential form $\omega \in \wedge^{\operatorname{dim} X} \Omega_{X}^{1} \otimes_{k}$ $k(X)$ (it depends on the choice of $\omega$, but only up to linear equivalence). Now $B$ is a finite combination of prime divisors with real non-negative coefficients, and the $\mathbb{R}$-Cartier hypothesis means that locally on $X, K_{X}+B$ equals a finite sum $\sum_{i} r_{i}\left(\varphi_{i}\right)$, where $r_{i} \in \mathbb{R}$ and $\varphi_{i} \in k(X)^{\times}$are non-zero rational functions on $X$.

Let $\mu: X^{\prime} \rightarrow X$ be a birational morphism. If we use the same top rational form to define canonical divisors, $K_{X}=(\omega)$ and $K_{X^{\prime}}=\left(\mu^{*} \omega\right)$, we have the log pull-back formula

$$
\mu^{*}\left(K_{X}+B\right)=K_{X^{\prime}}+B_{X^{\prime}} .
$$

As the notation suggests, the $\mathbb{R}$-Weil divisor $B_{X^{\prime}}$ is independent of the choice of $\omega$. For a prime divisor $E \subset X^{\prime}$, the real number $a(E ; X, B)=1-$ mult $_{E}\left(B_{X^{\prime}}\right)$ is called the $\log$ discrepancy of $(X, B)$ at $E$. This number depends only on the valuation of $k(X)$ defined by $E \subset X^{\prime} \stackrel{\mu}{\rightarrow} X$. We call such a valuation geometric, and denote $c_{X}(E)=\mu(E)$.

Definition 2.1. The $\log$ variety $(X, B)$ is said to have

- $\log$ canonical singularities if $a(E ; X, B) \geq 0$ for every geometric valuation $E$ of $X$.

- Kawamata log terminal singularities if $a(E ; X, B)>0$ for every geometric valuation $E$ of $X$.

The loci where $(X, B)$ has log canonical and Kawamata log terminal singularities, respectively, are non-empty open subsets of $X$. We denote their complements by $\operatorname{LS}(X, B)$ and $(X, B)_{-\infty}$, respectively. In particular, $(X, B)_{-\infty} \subseteq \operatorname{LCS}(X, B),(X, B)_{-\infty}=\emptyset$ if and only if $(X, B)$ has $\log$ canonical singularities, and $\operatorname{LCS}(X, B)=\emptyset$ if and only if $(X, B)$ has Kawamata log terminal singularities.

Remark 2.2. Suppose that $(X, B)$ does not have log canonical singularities. Then for every cycle $C \subseteq(X, B)_{-\infty}$ and every positive integer $n$, there exists a geometric valuation $E$ of $X$ such that $a(E ; X, B)<-n$ and $c_{X}(E)=C$. This property is behind our notation for the locus where $(X, B)$ does not have $\log$ canonical singularities.

Definition 2.3. A cycle $C \subset X$ is called a log canonical center if $(X, B)$ has log canonical singularities at the generic point of $C$, and there exists a geometric valuation $E$ of $X$ such that $a(E ; X, B)=0$ and $c_{X}(E)=C$.

Example 2.4. Let $X$ be a nonsingular variety and $B=\sum_{i=1}^{l} b_{i} E_{i}$, where $b_{i} \in \mathbb{R}_{\geq 0}$ and $\left\{E_{i}\right\}_{i=1}^{l}$ are nonsingular prime divisors intersecting transversely. Let $I_{0}=\left\{i ; b_{i}=1\right\}$ and $I_{-\infty}=\left\{i ; b_{i}>1\right\}$. Then 
- The locus where $(X, B)$ has Kawamata log terminal singularities is $X \backslash \cup_{i \in I_{0} \cup I_{-\infty}} E_{i}$. Its complement $\operatorname{LCS}(X, B)$ has a closed subscheme structure, with defining ideal

$$
\mathcal{O}_{X}\left(-\sum_{i \in I_{0} \cup I_{-\infty}}\left\lfloor b_{i}\right\rfloor E_{i}\right)
$$

- The locus where $(X, B)$ has log canonical singularities is the open set $X \backslash \cup_{i \in I_{-\infty}} E_{i}$.

- The log canonical centers of $(X, B)$ are the connected components of the intersections $\cap_{i \in J} E_{i}$, for $\emptyset \neq J \subseteq I_{0}$.

Remark 2.5. Our notion of log canonical center differs from the standard one used in the literature. The latter is defined as a center $C=c_{X}(E)$, where $E$ is a geometric valuation with $a(E ; X, B) \leq 0$. In our case, we further require that $(X, B)$ has log canonical singularities at the generic point of $C$. The two notions coincide for log varieties with $\log$ canonical singularities, but differ otherwise. For example, consider the log variety $\left(\mathbb{C}^{2}, H_{1}+2 H_{2}\right)$, where $H_{i}:\left(x_{i}=0\right)$. In the standard literature, $H_{1}, H_{2}$, and every point of $H_{2}$ is a $\log$ canonical center. In our sense, only $H_{1}$ is a log canonical center. This seems reasonable, given that $K_{\mathbb{C}^{2}}+H_{1}+2 H_{2}$ cannot be restricted by adjunction to $H_{2}$, or any its points. This also shows that the finiteness in Theorem 1.1.(1) fails in the non-log canonical case.

Remark 2.6. By Hironaka, we may choose $\mu$ so that $X^{\prime}$ is non-singular, and the proper transform $\mu_{*}^{-1} B$ and the $\mu$-exceptional locus $\cup_{i=1}^{l} E_{i}$ is supported by a simple normal crossings divisor. We have the following formula

$$
B_{X^{\prime}}=\mu_{*}^{-1} B+\sum_{i=1}^{l}\left(1-a\left(E_{i} ; X, B\right)\right) E_{i} .
$$

One can see that $(X, B)_{-\infty}$ is the image in $X$ of the components of $B_{X^{\prime}}$ with coefficients in $(1,+\infty)$, and $\operatorname{LCS}(X, B)$ is the image in $X$ of the components of $B_{X^{\prime}}$ with coefficients in $[1,+\infty)$. The lc centers of $(X, B)$ are the sets $\mu(S)$, where $S$ is a connected component of an intersection of components of $B_{X^{\prime}}$ with multiplicity one, such that $\mu(S) \nsubseteq(X, B)_{-\infty}$.

Remark 2.7. Canonical singularities were introduced by Reid (see [16]) as the singularities that appear on canonical models of projective manifolds of general type. Likewise, log canonical singularities are the singularities that appear on log canonical models of prototype log varieties of general type. It would be interesting to similarly describe semi-log canonical singularities (see [1]) and quasi-log canonical singularities (see [4]).

To see this in the log canonical case, let $(X, B)$ be a log variety with log canonical singularities. In the setting of Remark 2.6, the following formula holds:

$$
K_{X^{\prime}}+\mu_{*}^{-1} B+\sum_{i=1}^{l} E_{i}=\mu^{*}\left(K_{X}+B\right)+\sum_{i=1}^{l} a\left(E_{i} ; X, B\right) E_{i} .
$$

By $\log$ canonicity, $\sum_{i=1}^{l} a\left(E_{i} ; X, B\right) E_{i}$ is effective and $\mu$-exceptional, so this formula becomes a relative Zariski decomposition for the $\mu$-big log canonical divisor on the left-hand side, and we obtain

$$
\bigoplus_{m \in \mathbb{N}} \mu_{*} \mathcal{O}_{X^{\prime}}\left(m\left(K_{X^{\prime}}+\mu_{*}^{-1} B+\sum_{i=1}^{l} E_{i}\right)\right)=\bigoplus_{m \in \mathbb{N}} \mathcal{O}_{X}\left(m\left(K_{X}+B\right)\right)
$$


Further, if $B$ is rational, $(X, B)$ is recovered as follows: the graded $\mathcal{O}_{X}$-algebra on the left hand side is finitely generated (since the right-hand side is), its Proj is $X$, and $B$ is the push forward of $\mu_{*}^{-1} B+\sum_{i=1}^{l} E_{i}$ through the natural map $X^{\prime} \rightarrow$ Proj.

More generally, consider a $\log$ variety $\left(X^{\prime}, B^{\prime}\right)$ with $\log$ canonical singularities, and a proper morphism $\pi: X^{\prime} \rightarrow S$ such that $K_{X^{\prime}}+B^{\prime}$ is $\pi$-big and rational. We expect that the graded $\mathcal{O}_{S^{-}}$algebra $\mathcal{R}\left(X^{\prime} / S, B\right)=\bigoplus_{m \in \mathbb{N}} \pi_{*} \mathcal{O}_{X}\left(m\left(K_{X^{\prime}}+B^{\prime}\right)\right)$ is finitely generated. If so, we obtain a natural birational map $\Phi: X^{\prime} \rightarrow X:=\operatorname{Proj}\left(\mathcal{R}\left(X^{\prime} / S, B^{\prime}\right)\right)$, defined over $S$, and then $\left(X, \Phi_{*} B^{\prime}\right)$ has $\log$ canonical singularities.

\section{A TORSION FREENESS THEOREM}

The main result of this section is Theorem 3.4, the log canonical version of Kollár's torsion freeness [13]. It is a special case of [4, Theorem 3.2.(i)], but we reproduce it here for the convenience of the reader. We use vanishing results of Esnault-Viehweg [10, Theorem 5.1], based on logarithmic de Rham complexes. Recall that the characteristic is zero.

Theorem 3.1 ([10, Theorem 3.2). Let $X$ be a nonsingular projective variety and $T$ a $\mathbb{Q}$-divisor such that $T \sim_{\mathbb{Q}} 0$ and $T-\lfloor T\rfloor=\sum_{i=1}^{l} \delta_{i} E_{i}$ has simple normal crossings support. Let $d_{1}, \ldots, d_{l} \in \mathbb{Z}_{\geq 0}$, and $R$ a reduced divisor with no common components with $\sum_{i=1}^{l} E_{i}$, such that $R+\sum_{i=1}^{l} E_{i}$ has simple normal crossings. Denote $\mathcal{E}=\mathcal{O}_{X}(-R+\lfloor T\rfloor)$. Then the natural map of complexes

$$
\Omega_{X}^{\bullet}\left(\log R+\sum_{i=1}^{l} E_{i}\right) \otimes \mathcal{E}\left(-\sum_{i=1}^{l} d_{i} E_{i}\right) \rightarrow \Omega_{X}^{\bullet}\left(\log R+\sum_{i=1}^{l} E_{i}\right) \otimes \mathcal{E}
$$

is a quasi-isomorphism, and the spectral sequence

$$
E_{1}^{p q}=H^{q}\left(X, \Omega_{X}^{p}\left(\log R+\sum_{i=1}^{l} E_{i}\right) \otimes \mathcal{E}\right) \Longrightarrow \mathbb{H}^{p+q}\left(X, \Omega_{X}^{\bullet}\left(\log R+\sum_{i=1}^{l} E_{i}\right) \otimes \mathcal{E}\right)
$$

degenerates at $E_{1}$.

Sketch of proof. Let $n$ be a minimal positive integer with $n T \sim 0$. There exists a non-zero rational function $\varphi \in k(X)^{\times}$such that $(\varphi)=n T$. Let $\pi: X^{\prime} \rightarrow X$ be the normalization of $X$ in the field $k(X)(\sqrt[n]{\varphi})$. There exists an open subset $U \subseteq X$, with complement of codimension at least two, such that $U^{\prime}=\pi^{-1}(U)$ is nonsingular and the restriction to $U^{\prime}$ of the support $\sum_{i^{\prime}} E_{i^{\prime}}^{\prime}$ of $\pi^{*}\left(\sum_{i} E_{i}\right)$ is also a simple normal crossings divisor. Let $d: \mathcal{O}_{U^{\prime}} \rightarrow \Omega_{U^{\prime}}^{1}\left(\left.\log \sum_{i^{\prime}} E_{i^{\prime}}^{\prime}\right|_{U^{\prime}}\right)$ be the Kähler differential. The homomorphism $\pi_{*}(d)$ is compatible with action of the Galois group $\mathbb{Z} / n \mathbb{Z}=\langle\zeta\rangle$, so its component of eigenvalue $\zeta$, denoted $\nabla$, is an integrable connection on $\mathcal{O}_{X}(\lfloor T\rfloor)$ with logarithmic poles along $\sum_{i} E_{i}$. It is apriori defined only on $U$, but it extends to $X$ since $\mathcal{O}_{X}(\lfloor T\rfloor)$ is locally free. Its residues are

$$
\operatorname{Res}_{E_{i}}(\nabla)=\delta_{i} \cdot i d: \mathcal{O}_{X}(\lfloor T\rfloor) \otimes \mathcal{O}_{E_{i}} \rightarrow \mathcal{O}_{X}(\lfloor T\rfloor) \otimes \mathcal{O}_{E_{i}}
$$

Now $\nabla$ induces an integral connection on $\mathcal{O}_{X}(-R+\lfloor T\rfloor)$ with logarithmic poles along $R+\sum_{i} E_{i}$. Since the residue of this connection along each $E_{i}$ is given by multiplication with the fractional number $\delta_{j} \in(0,1)$, it follows from [10, Properties 2.9, Lemma 2.10] that the natural map of complexes is a quasi-isomorphism. The last statement follows from the degeneration of the spectral sequence associated to the Hodge filtration on a logarithmic 
de Rham complex (Deligne [5, Corollary 3.2.13]), applied to some desingularization of $X^{\prime}$.

Corollary 3.2 ([10], Theorem 5.1). Let L, D be Cartier divisors on a nonsingular projective variety $X$. Assume that there are nonsingular divisors $E_{i}$ intersecting transversely, and $b_{i} \in[0,1]$ such that:

(i) $L \sim_{\mathbb{R}} K_{X}+\sum_{i} b_{i} E_{i}$.

(ii) $D$ is effective, supported by $\sum_{0<b_{i}<1} E_{i}$.

Then the natural maps $H^{q}\left(X, \mathcal{O}_{X}(L)\right) \rightarrow H^{q}\left(X, \mathcal{O}_{X}(L+D)\right)$ are injective for all $q$.

Proof. The assumption (i) means that there exist rational functions $\varphi_{j} \in k(X)^{\times}$and $r_{j} \in \mathbb{R}$ such that $L=K_{X}+\sum_{i} b_{i} E_{i}+\sum_{j} r_{j}\left(\varphi_{j}\right)$. We fix $L-K_{X}-\sum_{b_{i} \in \mathbb{Q}} E_{i}$ and regard this equality of divisors as a system of equations in $b_{i} \in \mathbb{R} \backslash \mathbb{Q}$ and $r_{j}$, in the vector space whose basis consists of all the prime divisors involved. The space of solutions is defined over $\mathbb{Q}$, and since a solution exists, a rational solution exists. Therefore we may assume that $b_{i} \in \mathbb{Q}$ and $L \sim_{\mathbb{Q}} K_{X}+\sum_{i} b_{i} E_{i}$.

Denote $T=-L+K_{X}+\sum_{i} b_{i} E_{i}$. We have $T-\lfloor T\rfloor=\sum_{0<b_{i}<1} b_{i} E_{i}$ and $T \sim_{\mathbb{Q}} 0$. Denote $\mathcal{E}=\mathcal{O}_{X}\left(\left\lfloor-\sum_{b_{i}=1} E_{i}+T\right\rfloor\right)$ and consider the commutative diagram

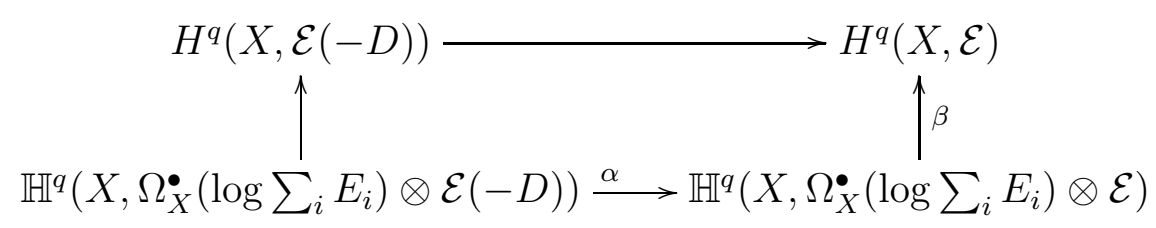

The first part of Theorem 3.1 gives that $\alpha$ is an isomorphism, and the second part implies that $\beta$ is surjective. We infer that $H^{q}(X, \mathcal{E}(-D)) \rightarrow H^{q}(X, \mathcal{E})$ is surjective. By Serre duality, $H^{0}\left(X, \omega_{X} \otimes \mathcal{E}^{-1}\right) \rightarrow H^{0}\left(X, \omega_{X} \otimes \mathcal{E}^{-1}(D)\right)$ is injective. This is the desired injective map, since $\omega_{X} \otimes \mathcal{E}^{-1}=\mathcal{O}_{X}\left(\left\lceil L-\sum_{0<b_{i}<1} b_{i} E_{i}\right\rceil\right)=\mathcal{O}_{X}(L)$.

Corollary 3.3. Let $\left(X, \sum_{i} b_{i} E_{i}\right)$ be a log variety such that $X$ is nonsingular and proper, $E_{i}$ are nonsingular divisors intersecting transversely, and $b_{i} \in[0,1]$ for all $i$. Let $L$ be a Cartier divisor on $X$, and $D$ an effective Cartier divisor, with the following properties:

(i) $H=L-\left(K_{X}+\sum_{i} b_{i} E_{i}\right)$ is a semiample $\mathbb{R}$-divisor. This means that $H=\sum_{i} h_{i} H_{i}$, where $h_{i} \geq 0$ and $\left|H_{i}\right|$ are linear systems free of base points.

(ii) $t H-D \sim_{\mathbb{R}} D^{\prime}$ for some effective $\mathbb{R}$-divisor $D^{\prime}$, and $t>0$.

(iii) $\operatorname{Supp}(D)$ and $\operatorname{Supp}\left(D^{\prime}\right)$ do not contain log canonical centers of $\left(X, \sum_{i} b_{i} E_{i}\right)$.

Then the natural maps $H^{q}\left(X, \mathcal{O}_{X}(L)\right) \rightarrow H^{q}\left(X, \mathcal{O}_{X}(L+D)\right)$ are injective for all $q$.

Proof. By Hironaka, there exists a birational modification $\mu: X^{\prime} \rightarrow X$ such that $X^{\prime}$ is projective and nonsingular, and $B_{X^{\prime}}=\mu^{*}\left(K_{X}+\sum_{i} b_{i} E_{i}\right)-K_{X^{\prime}}, \mu^{*} D$ and $\mu^{*} D^{\prime}$ are all suported by a simple normal crossings divisor $\sum_{i^{\prime}} E_{i^{\prime}}^{\prime}$. Decompose $B_{X^{\prime}}=B^{\prime}-A$ into the positive and negative part.

By assumption, $\mu^{*} D$ and $\mu^{*}\left(D^{\prime}\right)$ do not contain components of $\left\lfloor B^{\prime}\right\rfloor$. Therefore there exists $0<\epsilon \ll 1$ such that $\left\lfloor B^{\prime}+\lceil A\rceil-A+\epsilon \mu^{*} D+\epsilon \mu^{*}\left(D^{\prime}\right)\right\rfloor=\left\lfloor B^{\prime}\right\rfloor$. Since $\mu^{*} H$ is semiample, we may enlarge $\sum_{i^{\prime}} E_{i^{\prime}}^{\prime}$ and assume that there exists an $\mathbb{R}$-divisor $H^{\prime}$ with the following properties: $H^{\prime}$ is supported by $\sum_{i^{\prime}} E_{i^{\prime}}^{\prime}$, it has no common components with $B_{X^{\prime}}, \mu^{*} D$ and $\mu^{*}\left(D^{\prime}\right),\left\lfloor H^{\prime}\right\rfloor=0, H^{\prime} \sim_{\mathbb{Q}}(1-\epsilon t) \mu^{*} H$. We obtain

$$
\lceil A\rceil+\mu^{*} L \sim_{\mathbb{Q}} K_{X^{\prime}}+B^{\prime}+\lceil A\rceil-A+\epsilon \mu^{*} D+\epsilon \mu^{*}\left(D^{\prime}\right)+H^{\prime} .
$$


Since the effective Cartier divisor $\mu^{*} D$ is supported by the fractional part of the boundary $B^{\prime}+\lceil A\rceil-A+\epsilon \mu^{*} D+\epsilon \mu^{*}\left(D^{\prime}\right)+H^{\prime}$, Corollary 3.2 gives the injectivity of map

$$
H^{q}\left(X^{\prime}, \mathcal{O}_{X^{\prime}}\left(\lceil A\rceil+\mu^{*} L\right)\right) \rightarrow H^{q}\left(X^{\prime}, \mathcal{O}_{X^{\prime}}\left(\lceil A\rceil+\mu^{*} L+\mu^{*} D\right)\right) .
$$

On the other hand, $\mu_{*} \mathcal{O}_{X^{\prime}}(\lceil A\rceil)=\mathcal{O}_{X}$ and $R^{q} \mu_{*} \mathcal{O}_{X^{\prime}}(\lceil A\rceil)=0$ for $q>0$. In particular, $\mu_{*} \mathcal{O}_{X^{\prime}}\left(\lceil A\rceil+\mu^{*} L\right)=\mathcal{O}_{X}(L)$ and $R^{q} \mu_{*} \mathcal{O}_{X^{\prime}}\left(\lceil A\rceil+\mu^{*} L\right)=0$ for $q>0$. The Leray spectral sequence

$$
E_{2}^{p q}=H^{p}\left(X, R^{q} \mu_{*} \mathcal{O}_{X^{\prime}}\left(\lceil A\rceil+\mu^{*} L\right)\right) \Longrightarrow H^{p+q}\left(X^{\prime}, \mathcal{O}_{X^{\prime}}\left(\lceil A\rceil+\mu^{*} L\right)\right)
$$

degenerates, so we obtain an isomorphism $H^{q}\left(X, \mathcal{O}_{X}(L)\right) \stackrel{\sim}{\rightarrow} H^{q}\left(X^{\prime}, \mathcal{O}_{X^{\prime}}\left(\lceil A\rceil+\mu^{*} L\right)\right)$. Similarly, we obtain an isomorphism $H^{q}\left(X, \mathcal{O}_{X}(L+D)\right) \stackrel{\sim}{\rightarrow} H^{q}\left(X^{\prime}, \mathcal{O}_{X^{\prime}}\left(\lceil A\rceil+\mu^{*} L+\mu^{*} D\right)\right)$. The result follows.

Theorem 3.4. Let $\left(X, \sum_{i} b_{i} E_{i}\right)$ be a log variety such that $X$ is nonsingular, $E_{i}$ are nonsingular divisors intersecting transversely, and $b_{i} \in[0,1]$ for all $i$. Let $f: X \rightarrow S$ be a proper morphism, and $L$ a Cartier divisor on $X$ such that $L-K_{X}-\sum_{i} b_{i} E_{i}$ is $f$-semiample.

Let $q \geq 0$ and $s$ a local section of $R^{q} f_{*} \mathcal{O}_{X}(L)$, which is zero at the generic points of $f(X)$ and $f(C)$, for every log canonical center $C$ of $\left(X, \sum_{i} b_{i} E_{i}\right)$. Then $s=0$.

Proof. We may assume that $S$ is affine and $f(X)=S$. Then $L-K_{X}-\sum_{i} b_{i} E_{i}$ is semiample, and after possibly enlarging $\sum_{i} E_{i}$, we may assume $L \sim_{\mathbb{Q}} K_{X}+\sum_{i} b_{i} E_{i}$.

Assume by contradiction that the conclusion is false. Then there exists an effective very ample divisor $A$ on $S$ such that $f^{*} A$ does not contain any log canonical center of $\left(X, \sum_{i} b_{i} E_{i}\right)$, and the homomorphism $R^{q} f_{*} \mathcal{O}_{X}(L) \rightarrow R^{q} f_{*} \mathcal{O}_{X}(L) \otimes \mathcal{O}_{S}(A)$ is not injective. In particular, we may compactify $X$ and $S$ and assume that the homomorphism

$$
H^{0}\left(S, R^{q} f_{*} \mathcal{O}_{X}\left(L+f^{*} A\right)\right) \rightarrow H^{0}\left(S, R^{q} f_{*} \mathcal{O}_{X}\left(L+2 f^{*} A\right)\right)
$$

is not injective. Consider the commutative diagram of spectral sequences

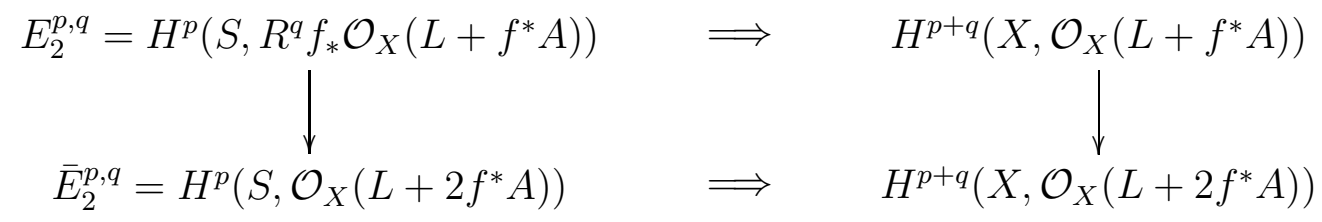

The map $E_{2}^{0, q} \rightarrow H^{q}\left(X, \mathcal{O}_{X}\left(L+f^{*} A\right)\right)$ is injective, so we conclude that the homomorphism

$$
H^{q}\left(X, \mathcal{O}_{X}\left(L+f^{*} A\right)\right) \rightarrow H^{q}\left(X, \mathcal{O}_{X}\left(L+2 f^{*} A\right)\right)
$$

is not injective. This contradicts Theorem 3.3 .

\section{Proof of Theorem 1.1}

Remark 4.1. Let $X$ be a nonsingular variety, $\sum_{i \in I} E_{i}$ a simple normal crossings divisor, $I^{\prime}, I^{\prime \prime}$ non-empty subsets of $I$, and $C$ a connected component of $\cap_{i \in I^{\prime}} E_{i}$. Then $C \subseteq \cup_{i \in I^{\prime \prime}} E_{i}$ if and only if $I^{\prime} \cap I^{\prime \prime} \neq \emptyset$.

Lemma 4.2. Let $(X, B)$ be a log variety with log canonical singularities, and $W$ a union of log canonical centers of $(X, B)$. Let $\mu: X^{\prime} \rightarrow X$ be a resolution of singularities with log pullback $\mu^{*}(K+B)=K_{X^{\prime}}+B_{X^{\prime}}$, such that $\mu^{-1}(W)$ is a divisor and $\mu^{-1}(W) \cup$ $\operatorname{Supp}\left(B_{X^{\prime}}\right)$ has simple normal crossings. Let $S$ be the union of prime divisors $E$ on $X^{\prime}$ with $\operatorname{mult}_{E}\left(B_{X^{\prime}}\right)=1$ and $E \subset \mu^{-1}(W)$. Then $\mathcal{O}_{W}=\mu_{*} \mathcal{O}_{S}$. 
Proof. We have a unique decomposition $B_{X^{\prime}}=S+R+\Delta-A$, where $S$ consists of the components of $B_{X^{\prime}}$ with coefficient equal to one and which are included in $\mu^{-1}(W), R$ consists of the components of $B_{X^{\prime}}$ with coefficient equal to one but which are not included in $\mu^{-1}(W), \Delta$ is the part of $B_{X^{\prime}}$ with coefficients in $(0,1)$ and $-A$ is the negative part of $B_{X^{\prime}}$. By Remark 4.1 applied to $R$ and $\mu^{-1}(W)$, no connected component of an intersection of components of $R$ is mapped inside $W$. Consider the exact sequence

$$
\mu_{*} \mathcal{O}_{X^{\prime}}(\lceil A\rceil) \rightarrow \mu_{*} \mathcal{O}_{S}\left(\left\lceil\left. A\right|_{S}\right\rceil\right) \rightarrow R^{1} \mu_{*} \mathcal{O}_{X^{\prime}}(\lceil A\rceil-S) .
$$

By $W=\mu(S),\lceil A\rceil-S=K_{X^{\prime}}+R+\lceil A\rceil-A+\Delta-\mu^{*}(K+B)$ and Theorem [3.4, the last map is zero. Therefore $\mu_{*} \mathcal{O}_{X^{\prime}}(\lceil A\rceil) \rightarrow \mu_{*} \mathcal{O}_{S}\left(\left.\lceil A\rceil\right|_{S}\right)$ is surjective. Since $B$ is effective, we deduce that $A$ is $\mu$-exceptional. Therefore $\mathcal{O}_{X}=\mu_{*} \mathcal{O}_{X^{\prime}}(\lceil A\rceil)$, which implies $\mu_{*} \mathcal{O}_{S}=\mathcal{O}_{W}$.

Proof of Theorem (1.1). (1) Choose $\mu: X^{\prime} \rightarrow X$ such that $X^{\prime}$ is smooth and $B_{X^{\prime}}$ has simple normal crossings support. The log canonical centers are the images on $X$ of the components of $B_{X^{\prime}}$ with coefficient one, and their intersections. Therefore they are finite.

(2) Let $C_{1}, C_{2}$ be two log canonical centers. By (1), it suffices to show that for every closed point $x \in C_{1} \cap C_{2}$, there exists a new log canonical center $x \in C_{3} \subset C_{1} \cup C_{2}$.

Let $W=C_{1} \cup C_{2}$. We may choose $\mu$ so that the hypotheses of Lemma 4.2 hold. In the notations of Lemma 4.2, we have $\mu_{*} \mathcal{O}_{S}=\mathcal{O}_{W}$. In particular, $S \rightarrow C_{1} \cup C_{2}$ has connected fibers. Therefore there are prime components $E_{1}, E_{2}$ of $S$ such that $\mu\left(E_{i}\right)=C_{i}$ and $E_{1} \cap E_{2} \cap \pi^{-1}(x) \neq \emptyset$. Let $Z$ be a connected component of $E_{1} \cap E_{2}$ which intersects $\pi^{-1}(x)$. Then $C_{3}=\mu(Z)$ is an log canonical center with $x \in C_{3} \subset C_{1} \cup C_{2}$.

(3) Let $W$ be the union of some $\log$ canonical centers. We may choose $\mu$ so that the hypotheses of Lemma 4.2 hold. In the notations of Lemma 4.2, we have $\mu_{*} \mathcal{O}_{S}=\mathcal{O}_{W}$. Since $S$ clearly has seminormal singularities, we infer by [2, Proposition 4.5] that $W$ has seminormal singularities.

(4) Fix $x \in \operatorname{LCS}(X, B)$ and consider $(X, B)$ as a germ near $x$. By (1) and (3), there exists a unique $\log$ canonical center $x \in C$ which is minimal with respect to inclusion. It remains to check that $C$ is normal near $x$. Construct $S$ as above with $\mu_{*} \mathcal{O}_{S}=\mathcal{O}_{C}$. Since $C$ is minimal, every connected component of an intersection of components of $S$ dominates $C$. Consider the simplicial scheme $\left(S_{n}=\left(S_{0} / S\right)^{\Delta_{n}} \rightarrow S\right)_{n \geq 0}$, where $S_{0} \rightarrow S$ is the normalization (see [6]). Each irreducible component of $S_{n}$ is nonsingular and mapped onto an intersection of components of $S$, hence it dominates $C$. Then each $S_{n} \rightarrow C$ factors through the normalization of $C$. These factorizations glue (cf. [4, Lemma 2.2.(ii)]), so that $S \rightarrow C$ factors through the normalization of $C$. But $\mu_{*} \mathcal{O}_{S}=\mathcal{O}_{C}$, so $C$ is normal.

\section{REFERENCES}

[1] Alexeev, V., Limits of stable pairs. preprint math.AG/0607684.

[2] Ambro, F., The locus of log canonical singularities. preprint math.AG/9806067.

[3] Ambro, F., The Adjunction Conjecture and its applications. PhD Thesis (February 1999), The Johns Hopkins University; math.AG/9903060.

[4] Ambro, F., Quasi-log varieties. Tr. Mat. Inst. Steklova 240 (2003), Biratsion. Geom. Linein. Sist. Konechno Porozhdennye Algebry, 220-239; translation in Proc. Steklov Inst. Math. 2003, no. 1 (240), 214-233.

[5] Deligne, P., Théorie de Hodge. II. Inst. Hautes Études Sci. Publ. Math. No. 40 (1971), 5-57.

[6] Deligne, P., Théorie de Hodge. III, Inst. Hautes Études Sci. Publ. Math, 44 (1974), 5-77. 
[7] Demailly, J.-P., Multiplier ideal sheaves and analytic methods in algebraic geometry. School on Vanishing Theorems and Effective Results in Algebraic Geometry (Trieste, 2000), 1-148, ICTP Lect. Notes, 6, Abdus Salam Int. Cent. Theoret. Phys., Trieste, 2001.

[8] Ein, L.; Lazarsfeld, R., Global generation of pluricanonical and adjoint linear series on smooth projective threefolds. J. Amer. Math. Soc. 6 (1993), no. 4, 875-903.

[9] Ein, L., Multiplier ideals, vanishing theorems and applications. Algebraic geometry-Santa Cruz 1995, 203-219, Proc. Sympos. Pure Math., 62, Part 1, Amer. Math. Soc., Providence, RI, 1997.

[10] Esnault, H.; Viehweg, E., Lectures on vanishing theorems. DMV Seminar, 20. Birkhäuser Verlag, Basel, 1992.

[11] Kawamata Y., Matsuda K., Matsuki K., Introduction to the minimal model program, Algebraic Geometry, Sendai, Advanced Studies in Pure Math. 10 (1987), 283-360.

[12] Kawamata, Y., On Fujita's freeness conjecture for 3-folds and 4-folds. Math. Ann. 308 (1997), no. $3,491-505$.

[13] Kollár, J., Higher direct images of dualizing sheaves. I. Ann. of Math. (2) 123 (1986), no. 1, 11-42.

[14] Kollár, J., Adjunction and discrepancies. In Flips and abundance for algebraic threefolds. Astérisque No. 211 (1992).

[15] Kollár, J. Singularities of pairs. Algebraic geometry-Santa Cruz 1995, 221-287, Proc. Sympos. Pure Math., 62, Part 1, Amer. Math. Soc., Providence, RI, 1997.

[16] Reid, M., Young person's guide to canonical singularities. Algebraic geometry, Bowdoin, 1985 (Brunswick, Maine, 1985), 345-414, Proc. Sympos. Pure Math., 46, Part 1, Amer. Math. Soc., Providence, RI, 1987.

[17] Shokurov, V. V., Three-dimensional log perestroikas. With an appendix in English by Yujiro Kawamata. Izv. Ross. Akad. Nauk Ser. Mat. 56 (1992), no. 1, 105-203; translation in Russian Acad. Sci. Izv. Math. 40 (1993), no. 1, 95-202.

[18] Siu, Y.-T., The Fujita conjecture and the extension theorem of Ohsawa-Takegoshi. Geometric complex analysis (Hayama, 1995), 577-592, World Sci. Publ., River Edge, NJ, 1996.

RIMS, Kyoto University, KYoto 606-8502, JAPAN.

E-mail address: ambro@kurims.kyoto-u.ac.jp 